\title{
Synbiotics use for preventing sepsis and necrotizing enterocolitis in very low birth weight neonates: a randomized controlled trial
}

\author{
Ozge Serce Pehlevan, MD ${ }^{1}$, Derya Benzer, MD ${ }^{1}$, Tugba Gursoy, $M D^{2}$, Guner Karatekin, MD ${ }^{1}$, Fahri Ovali, MD ${ }^{1}$ \\ ${ }^{1}$ Neonatology Unit, Zeynep Kamil Maternity and Children's Training and Research Hospital, University of Health Sciences, Istanbul, Turkey; ${ }^{2}$ Neonatology Unit, Koc \\ University School of Medicine, Istanbul, Turkey
}

\begin{abstract}
Background: Probiotics and prebiotics have strain-specific effects on the host. Synbiotics, a mixture of probiotics and prebiotics, are proposed to have more beneficial effects on the host than either agent has alone.

Purpose: We performed a randomized controlled trial to investigate the effect of Lactobacillus and Bifidobacterium together with oligosaccharides and lactoferrin on the development of necrotizing enterocolitis (NEC) or sepsis in very low birth weight neonates.

Methods: Neonates with a gestational age $\leq 32$ weeks and birth weight $\leq 1,500 \mathrm{~g}$ were enrolled. The study group received a combination of synbiotics and lactoferrin, whereas the control group received $1 \mathrm{~mL}$ of distilled water as placebo starting with the first feed until discharge. The outcome measures were the incidence of NEC stage $\geq 2$ or late-onset cultureproven sepsis and NEC stage $\geq 2$ or death.

Results: Mean birth weight and gestational age of the study $(n=104)$ and the control $(n=104)$ groups were $1,197 \pm 235 \mathrm{~g}$ vs. $1,151 \pm 269 \mathrm{~g}$ and $29 \pm 1.9$ vs. $28 \pm 2.2$ weeks, respectively $(P>0.05)$. Neither the incidence of NEC stage $\geq 2$ or death, nor the incidence of NEC stage $\geq 2$ or late-onset culture-proven sepsis differed between the study and control groups $(5.8 \%$ vs. $5.9 \%, P=1 ; 26 \%$ vs. $21.2 \%, P=0.51)$. The only significant difference was the incidence of all stages of NEC $(1.9 \%$ vs. $10.6 \%, P=0.019$ ).
\end{abstract}

Conclusion: The combination of synbiotics and lactoferrin did not reduce NEC severity, sepsis, or mortality.

Key words: Necrotizing enterocolitis, Sepsis, Synbiotics, Very low birth weight infant

\section{Key message}

Question: Can synbiotics, a combination of probiotics and prebiotics enhance beneficial effects in host more than either agent administered alone?
Finding: The combination of Lactobacillus and Bifidobacterium together with oligosaccharides and lactoferrin did not decrease the incidence of necrotizing enterocolitis or sepsis.

Meaning: As probiotics and prebiotics have strain-specific effects on the host, different combinations can be investigated for necrotizing enterocolitis or sepsis prophylaxis in future.

\section{Introduction}

Necrotizing enterocolitis (NEC) is characterized by dysbiosis of intestinal microbiota, barrier disruption, and exaggerated inflammation. ${ }^{1,2)}$ Systemic translocation of pathogen microorganisms to blood stream can occur when the barrier function of the gut is impaired. Preterm neonates have dysbiosis in gut microbiota secondary to antibiotic use, mode of delivery, and colonization from the hospital environment. ${ }^{1,3)}$ Unfortunately, current strategies to manage NEC are insufficient.

Functional foods are hope to reduce these diseases due to ability to modulate the composition and function of the intestinal microbiota. ${ }^{1,4)}$ Probiotics inhibits attachment of pathogenic bacteria competitively, decreases their colonization and translocation, and shifts the intestinal ecological balance from a potentially harmful microflora to one that is beneficial to the host. ${ }^{5)}$ Prebiotics stimulate the activity of probiotics. ${ }^{6}$ Although the Cochrane collaboration encourages the use of probiotics, there isn't sufficient evidence to change the practice. ${ }^{1,7)}$ Synbiotics, a combination of probiotics, and prebiotics, allow an increased function of exogenously administered probiotic microorganisms as well as providing substrate for endogenous commercial bacteria. ${ }^{1)}$ Lactoferrin, a prebiotic component in human milk, was reported to decrease late-onset sepsis (LOS) and NEC in low quality studies. ${ }^{2)}$

A combination of these agents should enhance more beneicial effects than when either agent is administered alone. Lately,

\footnotetext{
Corresponding author: Ozge Serce Pehlevan, MD. Neonatology Unit, Derince Training and Research Hospital, Alikahya, Kocaeli, Turkey

凶E-mail: ozge_serce@hotmail.com, https://orcid.org/0000-0001-5870-0630

Current affiliation - Ozge Serce Pehlevan: Neonatology Unit, Derince Training and Research Hospital, Kocaeli, Turkey; Fahri Ovall: Division of Neonatology, Department of Pediatrics, Istanbul Medeniyet University Medical Faculty, G ztepe, Istanbul

Received: 26 April, 2019, Revised: 10 December, 2019, Accepted: 24 December, 2019

This is an open-access article distributed under the terms of the Creative Commons Attribution Non-Commercial License (http://creativecommons.org/licenses/bync/4.0/) which permits unrestricted non-commercial use, distribution, and reproduction in any medium, provided the original work is properly cited.

Copyright (c) 2020 by The Korean Pediatric Society
} 


\begin{tabular}{|c|c|}
\hline $0.16(0.03-0.77)$ & Necrotizing enterocolitis (All stages) \\
\hline $0.56(0.47-0.67)$ & Stage $\geq 2$ Necrotizing enterocolitis \\
\hline $1.67(0.85-3.27)$ & Late onset culture proven sepsis \\
\hline $1.3(0.68-2.48)$ & Stage $\geq 2$ Necrotizing enterocolitis or late onset culture (+) sepsis \\
$0.99(0.30-3.17)$ & Stage $\geq 2$ Necrotizing enterocolitis or death \\
$0.57(0.49-8.39)$ & Deaths attributable to Stage $\geq 2$ Necrotizing enterocolitis \\
\hline $\mathbf{2 . 0 4}(\mathbf{0 . 4 9 - 8 . 3 9 )}$ & Deaths (all) \\
\hline
\end{tabular}

Graphical abstract. Relative risk of synbiotic administration.

the use of multistrain formulations are on the rise all around the world. ${ }^{8,9)}$ However, the host's response to different combination of synbiotics is variable. ${ }^{1)}$ Therefore, we aimed to evaluate the combined effect of Lactobacillus and Bifidobacterium together with oligosaccharides and lactoferrin on the development of NEC or LOS in very low birth weight (VLBW) neonates.

\section{Methods}

A prospective, double blinded, randomized, controlled trial (RCT) was conducted from February 2012, to September 2013, in Neonatal Intensive Care Unit (NICU). The study was approved by Ethical Committee of Zeynep Kamil Maternity and Children's Training and Research Hospital at February 27, 2012 (No. 16). This trial has been registered at (www.irct.ir; identifier number: IRCT2013062710279N3). VLBW infants (gestational age $\leq 32$ weeks; birth weight $\leq 1,500 \mathrm{~g}$ ) who survived to start feeding enterally were eligible for the trial. Infants who had severe asphyxia (stage III), major congenital anomalies, those who had fasted $\geq 3$ weeks, died in the first postnatal 14 days were excluded.

The recent incidence of primary outcomes was $31 \%$ for NEC or LOS, and 35\% for death or NEC in our NICU (NEC alone, $17 \%$; sepsis alone, $19 \%$; death alone, $18 \%)$. With the $\alpha$-error set at 0.05 and the $\beta$-error set at 0.2 , and an absolute reduction in the incidence of either NEC/LOS or NEC/death of 50\%, the number of infants needed to verify our hypothesis was 104 for NEC or LOS and 92 for NEC or death for each group. Family withdrawal was 11, and 19 infants died during the first 2 weeks after enrollment in our previous trial. ${ }^{3)}$ So we assumed that nearly 250 infants would be enough to be randomized by using sequential numbers generated at computer center. The infants were randomly assigned to the study or the control group after informed consent was obtained. The allocations were contained in opaque, sequentially numbered sealed envelopes. The study group received ISO-9001 certified combination of synbiotic and lactoferrin (Probiotic-ATP, Nobel, Turkey, 1/2 sachet every 12 hours); whereas the control group received placebo (distilled water; $1 \mathrm{~mL}$ every 12 hours) which were added to breast milk or formula (Aptamil Prematil Formula, Milupa-AG, Friedrichsdorf, Germany) starting with the first feed until discharge. The me- dian duration of synbiotic supplementation and follow-up period was 36 days. Synbiotic preparation contained 820 million Lactobacillus rhamnosus, 410 million L. plantarum, 410 million L. casei, 410 million Bifidobacterium lactis, 383-mg fructooligosaccharide (FOS), 100-mg galactooligosaccharide (GOS), 2-mg bovine lactoferrin, and vitamins (25-mg of vitamin C, 8-mg of vitamin E, 0.5-mg of vitamin B1, B2 and B6). The dosage of synbiotic supplementation was determined from previous studies. ${ }^{4)}$ The preparations, which were kept at room temperature and away from light and humidity, were added to breast milk or formula (Aptamil Prematil Formula) starting with the first feed until discharge. Following instructions from the sealed envelope, the personnel in the breast milk team mixed the supplements with distilled water to prepare a $1-\mathrm{mL}$ suspension and added it to the breast milk or formula before feeding; the addition of suspension did not change the appearance of the milk. Thus, the only personnel who knew of the infants' group assignments were one of the investigators (GT) and those in the breast milk team who were not involved in the care of the infants and data analyses. Minimal enteral feeding (10-20 mL/ $\mathrm{kg}$ ) was initiated on the first postnatal day if there was no contraindication (e.g., severe metabolic acidosis, asphyxia). A daily increase in the amount of feeding was based on birth weight $(10 \mathrm{~mL} / \mathrm{kg} /$ day for $<750 \mathrm{~g}, 20 \mathrm{~mL} / \mathrm{kg} /$ day for $750-1,250 \mathrm{~g}$, and $30 \mathrm{~mL} / \mathrm{kg} /$ day for 1,250-1,500 g birth weight). Feedings were withheld if there was gastric aspirate in an amount that was more than one-half of the previous feeding with abdominal distension or vomiting. Fortification was started when the enteral feeding reached $100 \mathrm{~mL} / \mathrm{kg} /$ day. A fixed dosage $(4 \mathrm{~g}$ per $100 \mathrm{~mL}$ human milk) of fortifier (Eoprotein, Milupa-AG) was added to the milk. The infants received total parenteral nutrition until 100 $\mathrm{mL} / \mathrm{kg} /$ day of enteral feeding was achieved. Infants with a birth weight below 2 standard deviation were classified as small for gestational age. ${ }^{10)}$ "Modified Bell Classification" was used to define NEC, and "Papille Classification" for intraventricular hemorrhage. ${ }^{11,12)}$ Bronchopulmonary dysplasia was defined in accordance with the definition provided by the National Instituted of Child Health and Human Development. ${ }^{13)}$ The culture proven LOS was defined in neonates who had clinical symptoms and laboratory signs and positive blood culture after 72 hours of life.

Demographic and clinical variables were recorded. The pri- 


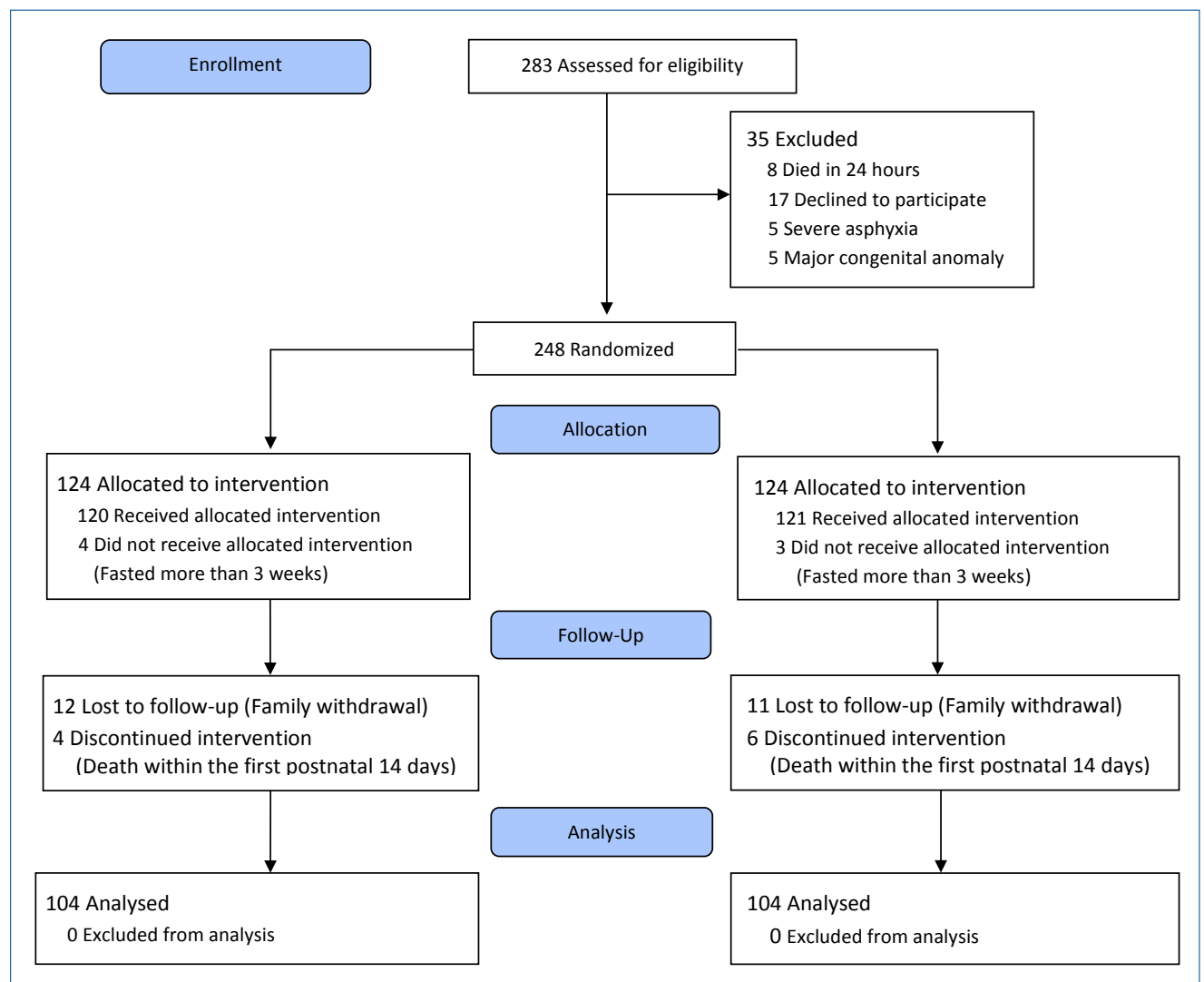

Fig. 1. Flow diagram of the study.

mary outcomes were stage $\geq 2$ NEC or culture proven LOS and stage $\geq 2$ NEC or death. Secondary endpoints were the time to reach $100 \mathrm{~mL} / \mathrm{kg} /$ day of enteral feeding, oxygen dependency at 36 weeks, mortality before hospital discharge, and duration of hospitalization. Adverse effects, including sepsis attributable to probiotic microorganisms, anaphylaxis and diarrhea were recorded.

Statistical analyses were performed by SPSS ver.13.0 (SPSS Inc., Chicago, IL, USA). Demographic data were stated as ratio, mean ( \pm standard deviation), and median (25th percentile75 th percentile). Dependent and independent variables were compared by chi-square and Fisher exact test. Independent samples $t$-test and Mann-Whitney $U$-test were used to compare continuous variables. Results were stated as relative risk ratio (RR) and 95\% confidence interval (CI). All statistical tests were 2 -sided, and a $P$ value of $\leq 0.05$ was considered to indicate statistical significance.

\section{Results}

Flow diagram of the study is shown in Fig. 1. Totally 283 VLBW neonates were admitted to NICU during the study period. Finally, 104 infants in each group completed the study. Demographic characteristics of the infants and clinical characteristics of the mothers did not differ between the groups (Table 1). The infants' clinical variables also did not differ between the groups, except for the increment rate of minimal enteral feeding
Table 1. Demographic and clinical characteristics of the mothers and infants

\begin{tabular}{lccc}
\hline Variable & $\begin{array}{c}\text { Control group } \\
(\mathrm{n}=104)\end{array}$ & $\begin{array}{c}\text { Synbiotic group } \\
(\mathrm{n}=104)\end{array}$ & $\begin{array}{c}P \\
\text { value }\end{array}$ \\
\hline Birth weight $(\mathrm{g})$ & $1,151 \pm 269$ & $1,197 \pm 235$ & 0.19 \\
Gestational age $(\mathrm{wk})$ & $28 \pm 2.2$ & $29 \pm 1.9$ & 0.10 \\
Cesarean section & $94(90.4)$ & $91(87.5)$ & 0.50 \\
APGAR, 5 minutes, median (range) & $7(68)$ & $7(68)$ & 0.59 \\
Male infant & $61(58.7)$ & $52(50.0)$ & 0.21 \\
PROM $>18 \mathrm{hr}$ & $23(22.1)$ & $19(18.3)$ & 0.49 \\
Preeclampsia & $33(31.7)$ & $33(31.7)$ & 1 \\
Prenatal steroid treatment & $81(77.9)$ & $85(81.7)$ & 0.49 \\
Small for gestational age & $17(16.3)$ & $12(11.5)$ & 0.35 \\
\hline
\end{tabular}

Values are presented as mean \pm standard deviation or number (\%) unless otherwise indicated.

PROM, prolonged rupture of the membranes.

(Table 2).

Table 3 shows the outcomes of the study. The only significant difference was determined in the incidence of all stages of NEC between the groups (11 [10.6\%] vs. 2 [1.9\%], $P=0.019)$. The incidence of stage $\geq 2 \mathrm{NEC}$ was $4(6.7 \%)$ in the control group and 0 in the study group $(P=0.13)$. Only one neonate who developed stage $\geq 2$ NEC died (25\%), and he was in the control group. Neither the incidence of stage $\geq 2$ NEC or death nor the incidence of stage $\geq 2$ NEC or culture proven LOS differed between the groups (Table 3).

Eighteen neonates (17.3\%) in the control group and 27 neonates $(26 \%)$ in the study group developed culture proven LOS. 
Of the microorganisms that grew, 55\% were gram positive and $45 \%$ were gram negative in the control group and $73 \%$ were gram positive and $27 \%$ were gram negative in the study group. No side effect attributed to the preparation (gastrointestinal intolerance or sepsis due to probiotics) was observed. However, towards the end of our study, we noticed that there was a concomitant vancomycin-resistant enterococcus (VRE) outbreak in NICU, but a causal relationship between the use of synbiotic preparation and this outbreak could not be established.

\section{Discussion}

This study could not demonstrate any reduction in the incidence of stage $\geq 2$ NEC or death nor the incidence of stage $\geq 2$ NEC or culture proven LOS with the concomitant use of probiotics and prebiotics. Well-designed trials of probiotics and prebiotics in NEC prophylaxis are required due to the heterogenity of available trials, and therefore this study is important. This combination could improve the survival of probiotic organism, as its specific substrate is readily available for its fermentation. However, the final effect of the interaction of each component of synbiotics on the host is the main question needed to be answered, as these agents may show additive or antagonistic

Table 2. Postnatal clinical characteristics of the infants

\begin{tabular}{|c|c|c|c|}
\hline Variable & $\begin{array}{l}\text { Control group } \\
\qquad(n=104)\end{array}$ & $\begin{array}{l}\text { Synbiotic group } \\
\quad(n=104)\end{array}$ & $\begin{array}{c}P \\
\text { value }\end{array}$ \\
\hline Respiratory distress syndrome & $30(28.8)$ & $16(15.4)$ & 0.19 \\
\hline Patent ductus arteriosus & $16(15.4)$ & $8(7.7)$ & 0.34 \\
\hline Intraventricular hemorrhage & $16(15.4)$ & $9(8.7)$ & 0.50 \\
\hline Bronchopulmonary dysplasia & $9(8.7)$ & $4(3.8)$ & 0.15 \\
\hline Start on MEF (day) & $2(12)$ & $2(1-2)$ & 0.88 \\
\hline Increment of MEF (mL/kg/day) & $20(15-20)$ & $20(20-20)$ & 0.014 \\
\hline$\%$ of Breast milk used ${ }^{\mathrm{a})}$ & $80(50-90)$ & $90(50-90)$ & 0.76 \\
\hline
\end{tabular}

Values are presented as number (\%) or median (interquartile range).

MEF, minimal enteral feeding.

${ }^{a}$ Median value of total amount of feeding from breast milk of each neonate during hospitalization.

Boldface indicates a statistically significant difference with $P<0.05$. effects. ${ }^{14)}$ Commonly used prebiotics like the ones used in our study include GOSs and FOSs, which are found in human milk. ${ }^{1)}$ We preferred to administer frequently used probiotic strains, which were also members of microbiota in healthy neonates, and were the effective strains in reducing mortality, NEC, LOS in meta-analyses. ${ }^{14)}$ Unfortunately, when we used their combination, we could not determine these beneficial effects. Cochrane collaboration supports the use of probiotics due to encouraging results of RCTs on NEC prophylaxis. ${ }^{15-17)}$ However, these studies used various strains which have great strain specific variability in function. ${ }^{7,15-19)}$ A combination of L. acidophilus and B. bifidum was reported to be more effective than either probiotic alone or a multistrain combination. ${ }^{20)}$ Therefore, the meta-analyses tried to identify strains with great efficacy. ${ }^{14)}$ The results showed reduction in mortality and morbidity only in a minority of the studied strains or combinations. Also, there was no clear overlap of certain strains which were significantly effective on multiple outcome domains. $\left.{ }^{14}\right)$ The authors concluded that these controversial results could be due to inadequate number or size of RCTs, or due to a true lack of effect for certain species. ${ }^{14)}$ The variability of strains or the doses of probiotics used in RCT's, as well as the diversity in their underlying mechanisms of action may explain the contradictory results of RCT's. Another study, which evaluated the effect of $B$. breve on NEC and sepsis also reported negative results. ${ }^{21)}$ The next hypothesis was that probiotics containing multiple strains might be the most effective ones in the prevention of NEC and mortality. The meta-analysis of Chang et al. ${ }^{22}$ showed that multiple strains probiotics were effective. On the other hand, van den Akker et al. ${ }^{14)}$ outlined interesting points at their meta-analyses. Both L. rhamnosus GG and B. lactis Bb-12/B94 appeared to be effective in reducing NEC. In addition, B. longum BB536 showed a similar trend. However, both the combination of L. rhamnosus GG with $B$. longum BB536, and the combination of $\mathrm{B}$. lactis $\mathrm{Bb}-12$ with $B$. longum BB536 showed no measurable effect. This may reflect an antagonistic effect of B. longum BB536 together with the other two strains. ${ }^{14)}$ This antagonism may also explain our negative results.

The prebiotics can directly promote intestinal cell barrier

Table 3. Study outcomes

\begin{tabular}{|c|c|c|c|c|}
\hline Variable & Control group $(n=104)$ & Synbiotic group $(n=104)$ & $P$ value & RR $(95 \% \mathrm{Cl})$ \\
\hline Time to reach $100 \mathrm{~mL} / \mathrm{kg} /$ day of oral feeding (day) & $10(7-15)$ & $10(6-14)$ & 0.23 & - \\
\hline Oxygen dependency at 36 wk PMA & $10(9.6)$ & $4(3.8)$ & 0.06 & $0.37(0.11-1.24)$ \\
\hline NEC (all stages) & $11(10.6)$ & $2(1.9)$ & 0.019 & $0.16(0.03-0.77)$ \\
\hline NEC (stage $\geq 2$ ) & $4(3.8)$ & $0(0)$ & 0.13 & $0.56(0.47-0.67)$ \\
\hline Late onset culture proven sepsis & $18(17.3)$ & $27(26)$ & 0.17 & $1.67(0.85-3.27)$ \\
\hline NEC (stage $\geq 2$ ) or late onset culture $(+)$ sepsis & $22(21.2)$ & $27(26)$ & 0.51 & $1.3(0.68-2.48)$ \\
\hline Stage $\geq 2$ NEC or death & $6(5.8)$ & $6(5.8)$ & 0.98 & $0.99(0.30-3.17)$ \\
\hline Deaths attributable to NEC (stage $\geq 2$ ) & $1(1.0)$ & $0(0)$ & 1.000 & $0.57(0.49-0.68)$ \\
\hline Deaths (all) & $3(2.9)$ & $6(5.8)$ & 0.49 & $2.04(0.49-8.39)$ \\
\hline Duration of hospitalization (day) & $39(26-56)$ & $36(26-52)$ & 0.44 & - \\
\hline
\end{tabular}

Values are presented as median (interquartile range) or number (\%).

$\mathrm{RR}$, relative risk; $\mathrm{Cl}$, confidence interval; $\mathrm{PMA}$, postmenstrual age; NEC, necrotizing enterocolitis.

Boldface indicates a statistically significant difference with $P<0.05$. 
integrity, and alter the epithelial cytokine expression as probiotics. ${ }^{23)}$ Therefore, the impact of prebiotics on NEC or sepsis prevention has been investigated. Unfortunately, the results of these limited RCT's are also confounding. ${ }^{5,24,25)}$ The main action of prebiotics is the stimulation of the growth and/or activity of probiotic microorganisms. Therefore, combining the prebiotics with probiotics in synbiotic formulation was believed to allow increased function of exogenously administered probiotic bacteria, and to provide substrate for endogenous commensal bacteria. Selection of specific prebiotic together with probiotics is a major concern at this point because the effect of prebiotics on probiotics may depend on their molecular structure. ${ }^{1)}$ Another disadvantage of using combined preparations can be the difficulty to predict the selectivity and specificity of each component and what the resulting mechanism of action of that combination will be. There are few studies investigating the effect of synbiotics in the management of NEC or sepsis. ${ }^{5,6,26-28)}$ An RCT, which used B. lactis and inulin, reported a lower incidence of NEC and mortality in both the probiotic and synbiotic arms, but no effect in prebiotic arm. ${ }^{5)}$ In this study, synbiotics did not have additive effect compared with the use of probiotics alone. The combination of $c$, and FOSs reduced the incidence and severity of NEC in another study. ${ }^{26)}$ Another RCT preferred to use a mixture, nearly similar to our preparation (8 strains of Lactobacillus, and Bifidobacterium in combination with FOSs), and did not show significant improvements in the severity of NEC, sepsis, or mortality, parallel to our results. $\left.{ }^{6}\right)$ Kadlec and Jakubec ${ }^{29)}$ showed that prebiotics had a direct effect on the adherence ability of certain probiotic strains while having no impact on other strains. Therefore, we should be careful about possible unpredictable host responses (synergistic, additive, or antagonistic effects) to synbiotics.

Lactoferrin has anti-inflammatory, immunomodulatory, and regenerative properties besides it's prebiotic activity. ${ }^{30)}$ Therefore, it is reasonable to include lactoferrin alone or combined with probiotics in prevention of NEC or sepsis. Although lactoferrin supplementation with or without probiotics was reported to decrease severe NEC in preterm infants in Cochrane review, the evidence was graded as low-to-moderate quality. ${ }^{2)}$ The optimal dosage of lactoferrin on the prevention of NEC is not clear. ${ }^{2)}$ Manzoni et al. ${ }^{30)}$ who demonstrated that bovine lactoferrin supplementation alone or in combination with $c$ reduced the incidence of of death-and/or severe NEC in VLBW neonates, suggested that dosages should be higher than $100 \mathrm{mg}$ a day. The low dose of lactoferrin $(2 \mathrm{mg})$ in our manufactured preparation when compared with the other RCT's should be taken into account when interpreting the results of our study. ${ }^{2)}$

There is always a theoretical risk of probiotic septicemia secondary to probiotics. there are few reports about Lactobacilli sepsis. ${ }^{31)}$ Lactobacillus GG sepsis observed in an extreme premature infant indicates that caution is required. ${ }^{32)}$ We did not observe any adverse events during the trial. However, towards the end of the study, we observed a VRE colonization outbreak in our NICU, though no sepsis case was detected. ${ }^{33)}$ Although the exact mechanism of this outbreak could not be determined, the resistance gene reservoir hypothesis suggests that commensal bacteria may play a major role in the transfer of antibiotic resistance to pathogenic bacteria. ${ }^{33)}$ This issue should be investigated further.

Main limitation of our study is that we can only interpret the results of combined effect of the mixture as we did not investigate the sole effect of each component on the incidence of NEC or sepsis. We also did not perform the stool culture for estimating the colony counts of Bifidobacteria and Lactobacillus.

In conclusion, the synbiotic mixture we used did not reduce the incidence of stage $\geq 2 \mathrm{NEC}$ or death nor the incidence of stage $\geq 2$ NEC or culture proven LOS. As the mechanism of action of different probiotic species' vary and is strain-specific, the effects of one probiotic strain can not be generalized to others. Further studies should be focused on different mixture of synbiotics due to the strain specific effects of probiotics, and complex interactions with each other in the host.

\section{Conflicts of interest}

Synbiotic preparations were supplied from Nobel pharmacy. No other potential conflicts of interest relevant to this study were reported.

\section{References}

1. Johnson-Henry KC, Abrahamsson TR, Wu RY, Sherman PM. Probiotics, prebiotics, and synbiotics for the prevention of necrotizing enterocolitis. Adv Nutr 2016;7:928-37.

2. Pammi M, Suresh G. Enteral lactoferrin supplementation for prevention of sepsis and necrotizing enterocolitis in preterm infants. Cochrane Database Syst Rev 2020; CD007137.

3. Serce O, Benzer D, Gursoy T, Karatekin G, Ovali F. Efficacy of Saccharomyces boulardii on necrotizing enterocolitis or sepsis in very low birth weight infants: a randomised controlled trial. Early Hum Dev 2013;89: 1033-6.

4. Deshpande GC, Rao SC, Keil AD, Patole SK. Evidence-based guidelines for use of probiotics in preterm neonates. BMC Med 2011;9:92.

5. Dilli D, Aydin B, Fettah ND, Özyazıcı E, Beken S, Zenciroğlu A, et al. The propre-save study: effects of probiotics and prebiotics alone or combined on necrotizing enterocolitis in very low birth weight infants. J Pediatr 2015;166:545-51.e1.

6. Nandhini LP, Biswal N, Adhisivam B, Mandal J, Bhat B V, Mathai B. Synbiotics for decreasing incidence of necrotizing enterocolitis among preterm neonates: a randomized controlled trial. J Matern Fetal Neonatal Med 2016;29:821-5.

7. Robinson J. Cochrane in context: probiotics for prevention of necrotizing enterocolitis in preterm infants. Evid Based Child Health 2014;9:672-4.

8. Härtel C, Pagel J, Rupp J, Bendiks M, Guthmann F, Rieger-Fackeldey E, et al. Prophylactic use of Lactobacillus acidophilus/Bifidobacterium infantis probiotics and outcome in very low birth weight infants. J Pediatr 2014;165:285-9.e1.

9. Duffield SD, Clarke P. Current use of probiotics to prevent necrotising enterocolitis. Arch Dis Child Fetal Neonatal Ed 2019;104:F228.

10. Ovali F. Intrauterine growth curves for Turkish infants born between 25 and 42 weeks of gestation. J Trop Pediatr 2003;49:381-3.

11. Walsh MC, Kliegman RM, Fanaroff AA. Necrotizing enterocolitis: a practitioner's perspective. Pediatr Rev 1988;9:219-26.

12. Papile LA, Burstein J, Burstein R, Koffler H. Incidence and evolution of 
subependymal and intraventricular hemorrhage: a study of infants with birth weights less than 1,500 gm. J Pediatr 1978;92:529-34.

13. Jobe AH, Bancalari E. Bronchopulmonary dysplasia. Am J Respir Crit Care Med 2001;163:1723-9.

14. van den Akker CH, van Goudoever JB, Szajewska H, Embleton ND, Hojsak I, Reid D, et al. Probiotics for preterm infants: a strain-specific systematic review and network meta-analysis. J Pediatr Gastroenterol Nutr 2018;67:103-22.

15. AlFaleh K, Anabrees J. Probiotics for prevention of necrotizing enterocolitis in preterm infants. Cochrane Database Syst Rev 2014; CD005496.

16. Deshpande G, Rao S, Patole S, Bulsara M. Updated meta-analysis of probiotics for preventing necrotizing enterocolitis in preterm neonates. Pediatrics 2010;125:921-30.

17. Wang Q, Dong J, Zhu Y. Probiotic supplement reduces risk of necrotizing enterocolitis and mortality in preterm very low-birth-weight infants: an updated meta-analysis of 20 randomized, controlled trials. J Pediatr Surg 2012;47:241-8.

18. Ofek Shlomai N, Deshpande G, Rao S, Patole S. Probiotics for preterm neonates: what will it take to change clinical practice? Neonatology 2014;105:64-70.

19. Mihatsch WA, Braegger CP, Decsi T, Kolacek S, Lanzinger H, Mayer B, et al. Critical systematic review of the level of evidence for routine use of probiotics for reduction of mortality and prevention of necrotizing enterocolitis and sepsis in preterm infants. Clin Nutr 2012;31:6-15.

20. Baucells BJ, Mercadal Hally M, Álvarez Sánchez AT, Figueras Aloy J. Probiotic associations in the prevention of necrotising enterocolitis and the reduction of late-onset sepsis and neonatal mortality in preterm infants under 1,500g: a systematic review. An Pediatr (Barc) 2016;85:247-55.

21. Costeloe K, Hardy P, Juszczak E, Wilks M, Millar MR; Probiotics in Preterm Infants Study Collaborative Group. Bifidobacterium breve BBG001 in very preterm infants: a randomised controlled phase 3 trial. Lancet 2016;387:649-60.

22. Chang HY, Chen JH, Chang JH, Lin HC, Lin CY, Peng CC. Multiple strains probiotics appear to be the most effective probiotics in the preven. tion of necrotizing enterocolitis and mortality: An updated meta-analysis. PLoS One 2017;12:e0171579.

23. Ortega-González M, Ocón B, Romero-Calvo I, Anzola A, Guadix E, Zarzuelo A, et al. Nondigestible oligosaccharides exert nonprebiotic ef- fects on intestinal epithelial cells enhancing the immune response via activation of TLR4-NFkB. Mol Nutr Food Res 2014;58:384-93.

24. Srinivasjois R, Rao S, Patole S. Prebiotic supplementation in preterm neonates: updated systematic review and meta-analysis of randomised controlled trials. Clin Nutr 2013;32:958-65.

25. Jantscher-Krenn E, Zherebtsov M, Nissan C, Goth K, Guner YS, Naidu $\mathrm{N}$, et al. The human milk oligosaccharide disialyllacto-N-tetraose prevents necrotising enterocolitis in neonatal rats. Gut 2012;61:1417-25.

26. Sreenivasa B, Sunil Kumar P, Suresh Babu MT, Ragavendra K. Role of synbiotics in the prevention of necrotizing enterocolitis in preterm neonates: a randomized controlled trial. Int J Contemp Pediatr 2015;2: 127-30.

27. D'Souza A, Cai CL, Kumar D, Cai F, Fordjour L, Ahmad A, et al. Cytokines and toll-like receptor signaling pathways in the terminal ileum of hypoxic/ hyperoxic neonatal rats: benefits of probiotics supplementation. Am J Transl Res 2012;4:187-97.

28. D'Souza A, Fordjour L, Ahmad A, Cai C, Kumar D, Valencia G, et al. Effects of probiotics, prebiotics, and synbiotics on messenger RNA expression of caveolin-1, NOS, and genes regulating oxidative stress in the terminal ileum of formula-fed neonatal rats. Pediatr Res 2010;67:52631.

29. Kadlec R, Jakubec M. The effect of prebiotics on adherence of probiotics. J Dairy Sci 2014;97:1983-90.

30. Manzoni P, Dall'Agnola A, Tomé D, Kaufman DA, Tavella E, Pieretto M, et al. Role of lactoferrin in neonates and infants: an update. Am J Perinatol 2018;35:561-5.

31. Whelan K, Myers CE. Safety of probiotics in patients receiving nutritional support: a systematic review of case reports, randomized controlled trials, and nonrandomized trials. Am J Clin Nutr 2010;91:687-703.

32. Meyer MP, Alexander T. Reduction in necrotizing enterocolitis and improved outcomes in preterm infants following routine supplementation with Lactobacillus GG in combination with bovine lactoferrin. J Neonatal Perinatal Med 2017;10:249-55.

33. Topcuoglu S, Gursoy T, Ovalı F, Serce O, Karatekin G. A new risk factor for neonatal vancomycin-resistant Enterococcus colonisation: bacterial probiotics. J Matern Fetal Neonatal Med 2015;28:1491-4. 